\title{
A case of fatal acute fatty liver of pregnancy and literature review
}

\author{
Irushna Antonette Perera ${ }^{1 *}$, Jebananthy Anandaselvam Pradeepan ${ }^{2}$, \\ Uruthirapasupathy Mayorathan ${ }^{3}$, Thirunavukarasu Kumanan ${ }^{2}$, \\ Kopalasuntharam Muhunthan ${ }^{4}$, Gowry Selvaratnam²
}

\author{
${ }^{1}$ Registrar in Medicine, ${ }^{2}$ Consultant Chest Respiratory Physician, University Medical Unit, Teaching Hospital Jaffna, \\ Jaffna, Sri Lanka \\ ${ }^{3}$ Consultant Judicial Medical Officer, Teaching Hospital Jaffna, Jaffna, Sri Lanka \\ ${ }^{4}$ Department of Obstetrics and Gynaecology, University Medical Unit, Teaching Hospital Jaffna, Jaffna, Sri Lanka
}

Received: 22 February 2018

Accepted: 28 March 2018

\section{*Correspondence: \\ Dr. Irushna Antonette Perera, \\ E-mail: irushna_p@yahoo.co.in}

Copyright: $@$ the author(s), publisher and licensee Medip Academy. This is an open-access article distributed under the terms of the Creative Commons Attribution Non-Commercial License, which permits unrestricted non-commercial use, distribution, and reproduction in any medium, provided the original work is properly cited.

\begin{abstract}
AFLP, though rare, is an obstetric emergency which carries a high incidence of maternal and perinatal mortality, despite optimal care. We report a case of a 23-year-old primi mother, who presented to us with vague symptoms of feeling unwell and abdominal pain, was diagnosed with AFLP, and was managed accordingly with the highest level of care available but succumbed a few days into her illness though timely delivery was able to save the baby. By this case report we once again wish to emphasize the value of a timely diagnosis with a high level of clinical suspicion and supportive laboratory investigations including imaging; the need for early termination of pregnancy; and adequate supportive care as the key management options for AFLP. Further, we wish to explore the current treatment options available for AFLP and discuss a few novel therapeutic strategies such as plasma exchange in treating such cases and the pros and cons associated with these treatment modalities.
\end{abstract}

Keywords: Acute fatty liver of pregnancy, Maternal mortality, Obstetric emergency, Plasma exchange

\section{INTRODUCTION}

Acute fatty liver of pregnancy(AFLP) is a rare, life threatening condition that occurs towards the latter part of pregnancy or in the early postpartum period with an incidence of 1 in 7000-16,000 pregnancies. ${ }^{1,2}$

Even though the exact pathogenesis is not evident, the etiology of AFLP is said to involve abnormalities in fetal hepatic mitochondrial fatty acid oxidation.

The process of Beta-oxidation of fatty acids in hepatic mitochondria is a complex sequence of events requiring several essential enzymes: mitochondrial trifunctional protein and its alpha-subunit, long-chain 3-hydroxyacylCoA dehydrogenase(LCHAD), are the two enzymes of this metabolic process, whose autosomally inherited genetic mutations are most closely associated with AFLP, especially the G1548C mutation of LCHAD. ${ }^{3}$

The differential diagnoses to be considered includes preeclampsia, infective hepatitis and cholestasis of pregnancy.

Careful history and physical examination, in conjunction with compatible laboratory and imaging results, are often sufficient to make the presumptive diagnosis, and liver biopsy is usually not indicated.

Prompt delivery of the fetus and supportive management of liver failure are the key measures for optimal maternal and fetal outcome in AFLP. 


\section{CASE REPORT}

A 23-year-old primi mother was transferred to our tertiary care institution from a peripheral hospital with abdominal pain, nausea and vomiting for about 6 hours duration at a POA of 33 weeks.

Her booking visit was at 14 weeks and the antenatal follow up was regular. Her physical examination was unremarkable on admission apart from bilateral pitting pedal edema, and since she had no signs of onset of labour, was kept under close monitoring.

Twelve hours into the admission she developed spontaneous rupture of membranes with an unfavorable cervix and meconium stained liquor.

As she also recorded a pathological Cardiotocogram, an emergency Lower Segment Caesarian section (LSCS) was performed and a baby girl (birth weight $2.7 \mathrm{~kg}$ ) was delivered with an APGAR of 5 at birth and 8 at 5 minutes.

Intraoperatively about $500 \mathrm{ml}$ of free fluid was noted in the abdomen, and on careful evaluation the mother was found to be icteric.

Unexpectedly she had a post-partum haemorrhage of approximately $1000 \mathrm{ml}$ and it was managed according to standard protocols and was hemodynamically stabilized before transferring to the obstetric ICU for close monitoring.

A FBC performed on admission revealed a WBC 27, 650/cumm, Hemoglobin 7.1 g/dl, platelets 120,000/cumm, Prothrombin time $17.5 \mathrm{sec}$ with an INR of 1.46, ALT 68 $\mathrm{U} / \mathrm{L}$ and AST $70 \mathrm{U} / \mathrm{L}$, ALP 440U/L and a CRP of $33 \mathrm{mg} / \mathrm{L}$.

A presumptive diagnosis of HELLP syndrome or AFLP was made. Overwhelming infection on a preexisting liver condition was also entertained as a differential diagnosis. An USS of the abdomen confirmed a normal sized hypoechoic liver with free fluid in the abdomen.

Her liver functions continued to deteriorate with worsening coagulopathy and cholestasis. Surprisingly her transaminase levels remained well within normal limits. She received supportive care for an acute hepatic failure and expert opinion was sought from hepatologists from specialized centers in the island.

Though the coagulopathy was contained with daily transfusion of fresh frozen plasma and vitamin $\mathrm{K}$, the cholestasis was worsening by the day with increasing jaundice and hepatic encephalopathy was ensuing with a deteriorating level of consciousness.

Episodes of hypoglycemia was managed with regular blood sugar monitoring and treatment with intravenous
Dextrose. Broad spectrum intravenous antibiotics were continued to combat the concomitant overwhelming sepsis as evidenced by a rising CRP, which was at $82.9 \mathrm{mg} / \mathrm{L}$ on day 08 after admission. Ursodeoxycholic acid was also initiated during the course to treat the worsening cholestasis. N-acetylcysteine (NAC) infusion was started as a hepatoprotective measure and plasma exchange was initiated on post-partum day 08 .

With respiratory failure setting in, hypoxia worsened despite of high flow oxygen, and she was initially managed with Continuous positive airway pressure ventilation(CPAP) and later was ventilated invasively.

Worsening hepatic encephalopathy was noted and cerebral edema was confirmed by a NCCT brain. Her urine output slowly regressed, with rising serum creatinine, and the Hepatorenal syndrome was managed by renal replacement therapy in the form of intermittent hemodialysis.

The second cycle of plasmapheresis was done on the following day, but without a significant improvement in her clinical condition.

Despite of maximal supportive care she succumbed to her illness on day 10 post-partum. Autopsy showed marked icterus all over the body. Abdominal cavity contained $800 \mathrm{ml}$ of blood stained ascites.

The liver was found to be enlarged and measured $1.8 \mathrm{~kg}$. Surface and cut sections of the liver revealed greenish yellow discoloration due to fat infiltration and intrahepatic cholestasis (Figure1).

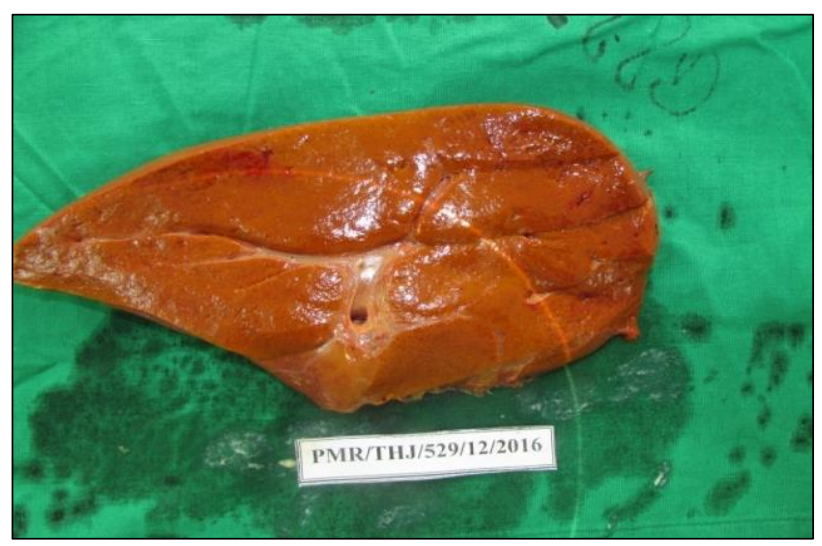

Figure 1: Surface/cut sections of the liver revealed greenish yellow discoloration.

Brain was edematous, and the lungs showed areas of small consolidations. The kidneys were swollen with altered cortico-medullary pattern.

Histology of the liver revealed a preserved architecture with micro and macro vesicular steatosis of parenchymal cells. Micro vesicular steatosis was predominant involving cells of the periportal area (Figure 2). Sinusoidal dilatation was evident and lobular 
inflammation was minimal. There was no interface hepatitis.

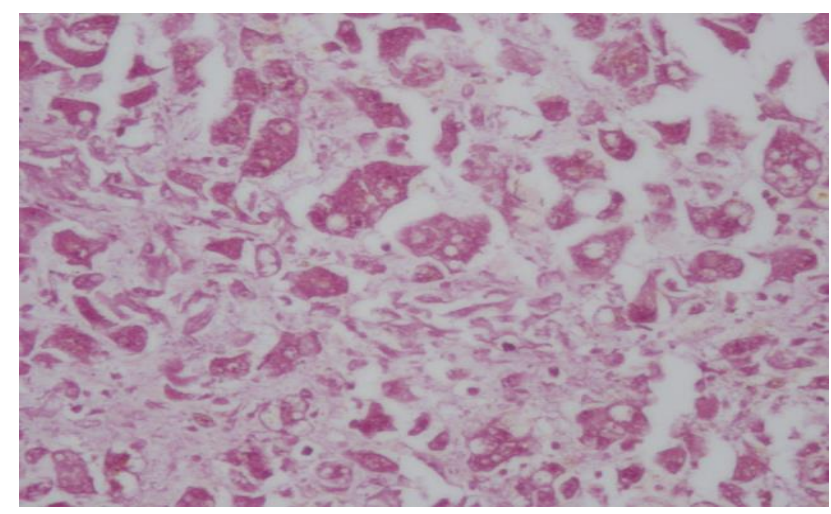

Figure 2: Micro and macro vesicular steatosis of liver parenchymal cells.

Lobar pneumonia and edema was noted in the lungs and kidneys showed acute tubular necrosis. With timely intervention we could save the baby, but the mother failed to respond to all the supportive measures taken to combat the multi organ dysfunction that had set in.

\section{DISCUSSION}

Acute Fatty liver of pregnancy(AFLP), is a disease entity unique to pregnancy, which is usually encountered towards the third trimester. The earliest literature available on AFLP, dates back to 1940, as described by Sheehan as an," Acute yellow atrophy of the liver", following which there have been many reported cases of the disease and its outcome. ${ }^{4}$

The pathogenesis, still poorly understood, is postulated to be an abnormality in the metabolism of long chain fatty acids in the fetus, where a deficiency in the enzyme longchain 3-hydroxyacyl-CoA dehydrogenase(LCHAD), will lead to an excess of fetal long chain fatty acids entering the maternal circulation, overwhelming the capacity of the maternal liver to handle long chain fatty acids, resulting in their deposition in the maternal liver and ultimately culminating in hepatic failure. ${ }^{5}$

AFLP, though rare, continues to be a life-threatening condition to date, and is usually seen to occur around the 36th week of gestation. ${ }^{6}$

Proposed risk factors in developing AFLP, include primiparity, pregnancy with a male fetus, multiple gestations, advanced maternal age, and low body mass index of the mother. ${ }^{7}$ The condition is neither infectious nor inherited. ${ }^{8}$ Also, the recurrence of the disease in a subsequent pregnancy is said to be very rare. ${ }^{9}$

The clinical presentation of AFLP is usually with non specific symptoms, but the possibility of AFLP should be considered in all pregnant women presenting with symptoms such as anorexia, nausea, vomiting, headache, fatigue and abdominal pain, towards the latter part of the 2nd trimester or in the 3rd trimester, as cases have been reported in as early as 22 weeks of gestation. ${ }^{10}$

The important differential diagnoses to be considered are fulminant viral hepatitis and severe HELLP syndrome. The latter is usually encountered in the setting of severe pre-eclampsia, and the frequent accompaniment of hypoglycemia and a prolonged prothrombin time favours a diagnosis of AFLP. Also, histology findings differ in these two conditions, with prominent periportal hemorrhages and fibrin deposition being characteristic of HELLP syndrome, while micro vesicular fatty infiltration favours AFLP. ${ }^{11}$

Cholestasis of pregnancy is another disease condition, unique to pregnancy, occurring in the 3rd trimester, which classically presents with pruritus, jaundice, with minimally elevated transaminases and a markedly elevated ALP level. It is usually considered as a benign and self-limiting condition but may be associated with adverse fetal outcomes. ${ }^{12}$

The usual laboratory investigation abnormalities seen in AFLP, include an elevated international normalized ratio (INR), a modest elevation in serum transaminases, an elevated bilirubin level, and thrombocytopenia with or without disseminated intravascular coagulation (DIC). Hyperuricemia, along with acute kidney injury (AKI) has also been reported in severe cases.13 Although the diagnosis can be confirmed by a liver biopsy, this is generally not encouraged in all cases, as the clinical evidence remains adequate in many instances, and also due to the accompanying coagulopathy with risk of intraabdominal bleeding. ${ }^{11}$ Ultra sound scanning remains the imaging modality of choice being both safe and convenient.

The disease, if not intervened early, may be complicated with upper gastro-intestinal bleeding due to altered coagulation profile, AKI, infection, pancreatitis or hypoglycemia. ${ }^{11}$ As a result of early diagnosis and intervention, the worldwide maternal mortality recorded as $100 \%$ in the past, have now reached rates $<10 \%$. But on the contrary, the fetal mortality remains high around $20 \% .^{14}$

A recent multicenter retrospective study conducted in China, has reported an association of a male fetus, postpartum diagnosis of AFLP (as in the case of our patient), intrauterine fetal death, DIC, altered Prothrombin time(PT) and activated partial thromboplastin time(APTT) as potential factors influencing maternal outcomes. ${ }^{15}$

The diagnosis of AFLP remains largely clinical, and the clinical diagnosis of AFLP is according to the "Swansea criteria", which is a collection of symptoms, signs, 
biochemical and imaging findings, where the presence of six or more of its components, in the absence of another cause, helps in establishing a clinical diagnosis of AFLP (Table 1). ${ }^{16}$

Table 1: Swansea criteria for the diagnosis of AFLP.

\begin{tabular}{|c|c|}
\hline Clinical & $\begin{array}{l}\text { Vomiting } \\
\text { Abdominal pain } \\
\text { Polydipsia/polyuria } \\
\text { Encephalopathy }\end{array}$ \\
\hline \multirow[t]{3}{*}{ Biochemical- Hepatic } & Bilirubin $>14 \mu \mathrm{mol} / \mathrm{l}$ \\
\hline & AST/ALT >42 IU/1 \\
\hline & Ammonia $>47 \mu \mathrm{mol} / 1$ \\
\hline \multirow[t]{2}{*}{ Renal } & Urate $>340 \mu \mathrm{mol} / \mathrm{l}$ \\
\hline & Creatinine $>150 \mu \mathrm{mol} / \mathrm{l}$ \\
\hline Endocrine & Glucose $<4 \mathrm{mmol} / \mathrm{l}$ \\
\hline \multirow[t]{2}{*}{ Hematological } & Leucocytosis $>11 \times 10^{9} / 1$ \\
\hline & $\begin{array}{l}\text { Coagulopathy-PT }>14 \text { secs } \\
\text { or } \\
\text { APTT }>34 \text { secs } \\
(\text { often with Plt count }>100 \\
\left.\times 10^{12} / 1\right)\end{array}$ \\
\hline Radiological & $\begin{array}{l}\text { Abdominal USS bright Liver } \\
\text { echo texture /ascites }\end{array}$ \\
\hline $\begin{array}{l}\text { Histological-Liver } \\
\text { Biopsy }\end{array}$ & Micro vesicular steatosis \\
\hline
\end{tabular}

Our patient described in the case report fulfilled almost all the criteria to be diagnosed with AFLP, apart from the serum Ammonia level, which was not performed, and the post mortem histology was able to confirm the presence of micro vesicular steatosis of the liver.

The accepted and currently recommended mode of treatment of this catastrophic disease entity remains the prompt delivery of the fetus, following the diagnosis of AFLP as early diagnosis and intervention has been shown to result in better maternal as well as fetal outcomes. ${ }^{7,17-19}$ Termination of the pregnancy may be in the form of induced labour, but generally caesarian section is the better option, as the mortality rate of mothers who undergo Lower segment caesarian section (LSCS), is shown to be lower than for those who deliver vaginally.18 Intensive supportive treatment and continuous close monitoring in the immediate postpartum period remains equally important for a successful outcome, and patients who remain critically ill should ideally be managed at tertiary care hospitals with a multidisciplinary team involving intensivists, obstetrician, hepatologist, neonatologist, and if indicated the organ transplant unit. ${ }^{20}$ Mortality is attributed to complications of the disease in the form of Acute respiratory distress syndrome (ARDS), hepatic failure, renal insufficiency, sepsis, DIC, encephalopathy, haemorrhagic shock either due to intra-abdominal bleeding or upper gastrointestinal haemorrhage, finally culminating in multi organ dysfunction. In the setting of fulminant hepatic failure, liver transplantation remains to be the last resort.
$\mathrm{N}$-acetylcysteine(NAC), a precursor of Glutathione, has also shown some success in patients with NonAcetaminophen-induced Acute liver failure(NAI-ALF), attributed to its anti-inflammatory, anti-oxidant, inotropic and vasodilatory effects which improve the microcirculation and oxygenation of vital organs, providing a survival benefit, as reported by Mumtaz K. et al following a prospective study of 47 patients with NAIALF. ${ }^{21}$

Novel treatment modalities such as plasmapheresis and the use of activated protein $\mathrm{C}$ have been practiced in specialized centers, with variable outcomes. ${ }^{22-24}$ According to a report by R.S.M Mohommad et al, on 3 cases of AFLP complicated with multiorgan failure, treated with repeated sessions of plasmapheresis, the results look promising, but this modality of treatment still needs to be explored in detail. Plasmapheresis appears to be a promising treatment option in managing patients with AFLP,as it replaces the function of the liver in part, by removal of ammonia, endotoxins, bilirubin, and inflammatory cytokines from the maternal circulation. Also, the timing of initiation of plasma exchange seems to play an important role in altering the course of the disease as shown by a study of 39 patients with acute fatty liver of pregnancy, as reported by Jin F, et al. ${ }^{22}$

In conclusion, through this case report we wish to reemphasize the grave nature of this disease entity which is unique to pregnancy, the importance of early detection and intervention and the need for more promising modalities of treatment in managing severe intractable cases.

\section{ACKNOWLEDGMENTS}

We sincerely wish to thank all the members of the staff of Teaching hospital Jaffna who helped us in the management of this patient, and in carrying out the diagnostic and therapeutic procedures and all those who helped us in preparation of this case report.

Funding: No funding sources Conflict of interest: None declared

Ethical approval: Not required

\section{REFERENCES}

1. Castro MA, Fassett MJ, Reynolds TB, Shaw KJ, Goodwin TM. Reversible peripartum liver failure: a new perspective on the diagnosis, treatment, and cause of acute fatty liver of pregnancy, based on 28 consecutive cases. Am J Obstet Gynecol. 1999; 181(2):389-95.

2. Reyes H, Sandoval L, Wainstein A, Ribalta J, Donoso S, Smok G, Rosenberg H, Meneses M. Acute fatty liver of pregnancy: a clinical study of 12 episodes in 11 patients. Gut. 1994;35(1):101-6.

3. Shames BD, Fernandez LA, Sollinger HW, Chin LT, D'Alessandro AM, Knechtle SJ, Lucey MR, Hafez R, 
Musat AI, Kalayoglu M. Liver transplantation for HELLP syndrome. Liver Transpl. 2005;11(2):224-8.

4. Hin Hin Ko, Eric Yoshida. Acute fatty liver of pregnancy. Can J Gastroenterol. 2006;20(1):25-30.

5. Martin S. Fatty Liver of Pregnancy. Clinical advisor; Obstetrics and Gynecology. 2017. Available at https://www.cancertherapyadvisor.com/obstetricsand-gynecology/fatty-liver-ofpregnancy/article/618207/

6. Tran TT, Ahn J, Reau NS. ACG Clinical Guideline: Liver Disease and Pregnancy. Am J Gastroenterol. 2016;111(2):176-94.

7. Ablett J. Acute fatty liver of pregnancy guideline (GL780). Maternity Guidelines- Acute Fatty Liver of Pregnancy (GL780). 2016;1-5

8. Brooks RR, Feller CM, Maye JP. Acute fatty liver of pregnancy: A case report. AANA Journal. 2002 ;70(3):215-7.

9. Bacq Y. Acute fatty liver of pregnancy. Semin Perinatol. 1998;22(2):134-40.

10. Trang, H. and Tuyet, H. (2017) Case Report: Acute Fatty Liver of Pregnancy. Open J Obstet Gynecol. 2017;7(10):1017-23.

11. Ibdah JA. Acute fatty liver of pregnancy: an update on pathogenesis and clinical implications. World J Gastroenterol. 2006;12(46):7397-404.

12. Pusl T, Beuers U. Intrahepatic cholestasis of pregnancy. Orph J Rare Dis. 2007;2(1):26.

13. Gilbert S, Weiner D. the Kidney in pregnancy: Section 8- Tubulointerstitial Nephropathies and disorders of the urinary tract. National Kidney Foundation Primer on Kidney diseases. 6th edition. Philadelphia. 2014:394.

14. Zaman A. Acute fatty liver disease of pregnancy: a review. Am J Gastroenterol. 2017.

15. Gao Q, Qu X, Chen X, Zhang J, Liu F, Tian S, Wang C. Outcome and risk factors of patients with acute fatty liver of pregnancy: a multicentre retrospective study. Singapore Med J. 2018.

16. Cowie P, Johnston IG. Acute fatty liver of pregnancy. Anaesthesia tutorial of the week. 2010.
17. Wei Q., Zhang L, Liu X.Clinical diagnosis and treatment of acute fatty liver of pregnancy: A literature review and 11 new cases. J Obstet Gynaecol Res. 2010;36(4):751-6.

18. Dwivedi S, Runmei M. Retrospective Study of Seven Cases with Acute Fatty Liver of Pregnancy. ISRN Obstetrics and Gynecology, vol. 2013, Article ID 730569, 7 pages, 2013.

19. Yun-Yan Chen,Li-Ying Gu, Wen Di,Jian-Hua Lin. Acute fatty liver of pregnancy: a retrospective analysis of 13 cases. Int J Clin Exp Pathol. 2017;10(6):7075-81.

20. Pandey CK, Karna ST, Pandey VK, Tandon M. Acute liver failure in pregnancy: Challenges and management. Indian J. Anaesth. 2015;59(3):144-9.

21. Mumtaz K, Azam Z, Hamid S, et al. Role of Nacetylcysteine in adults with non-acetaminopheninduced acute liver failure in a center without the facility of liver transplantation. Hepatology Int. 2009;3(4):563-70.

22. Seyyed Majidi MR, Vafaeimanesh J. Plasmapheresis in Acute Fatty Liver of Pregnancy: An Effective Treatment. Case Reports in Obstetrics and Gynecology. 2013;2013:615975.

23. Jin F, Cao M, Bai Y, Zhang Y, Yang Y, Zhang B. Therapeutic effects of plasma exchange for the treatment of 39 patients with acute fatty liver of pregnancy. Discov Med. 2012;13(72):369-73.

24. MacLean AA, Almeida Z, Lopez P. Complications of acute fatty liver of pregnancy treated with activated protein C. Arch Gynecol Obstet. 2005;273(2):119-21.

Cite this article as: Perera IA, Pradeepan JA, Mayorathan U, Kumanan T, Muhunthan K, Selvaratnam G. A case of fatal acute fatty liver of Pregnancy and Literature Review. Int J Reprod Contracept Obstet Gynecol 2018;7:2026-30. 